\title{
Esophageal cancer treated by endoscopic submucosal dissection after neoadjuvant chemoradiotherapy: a case report
}

\author{
Jang Hwan Lim, Sang Ah Lee, Gyoun Eun Kang, Jong Min Kim, Jin Kyung Park, Yun Jin Chung, Jae Kwon Jung, \\ Chang Keun Park, Hyun Soo Kim and Dong Wook Lee
}

\begin{abstract}
Introduction: The treatment of esophageal cancer remains clinically challenging because of the overall poor prognosis associated with the disease. The mortality rate associated with surgical treatment is high, and the majority of diagnosed patients are old. As such, surgery is not possible in many cases, even when the cancer has progressed to a resectable state.

Case presentation: We present the case of an 82-year-old Korean man who presented to our institution with intermittent odynophagia. Esophageal cancer with submucosal invasion and metastasis to three regional lymph nodes was diagnosed. After neoadjuvant chemoradiotherapy, his regional lymph nodes disappeared. Because of his poor pulmonary function, surgical treatment could not be performed. Endoscopic submucosal dissection was carried out instead, and endoscopic triamcinolone injections were performed serially. Neither recurrence nor abnormal symptoms such as dysphagia or regurgitation have developed for 36 months.
\end{abstract}

Conclusions: The literature suggests that endoscopic submucosal dissection after chemoradiotherapy is a viable treatment modality in patients with esophageal cancer with a high surgical treatment risk.

Keywords: Endoscopic submucosal dissection, Esophageal cancer, Neoadjuvant chemoradiotherapy

\section{Introduction}

Esophageal cancer is a highly lethal malignancy with a 5 -year survival rate of approximately $15 \%$ [1]. Thus, the management of patients with esophageal cancer is challenging and requires a multimodal approach. Suboptimal results have been reported for post-operative adjuvant chemotherapy [2], radiotherapy [3] and concurrent chemoradiotherapy [4]; thus, interest in neoadjuvant treatment has recently increased. In particular, Sjoquist et al. reported that, among patients with resectable esophageal cancer, those who received neoadjuvant chemoradiotherapy showed a significant survival benefit compared to patients who received surgical treatment alone [5]. However, the incidence of esophageal cancer and squamous cell carcinoma is known to increase with age. The

\footnotetext{
* Correspondence: storm5333@naver.com

Division of Gastroenterology and Hepatology, Department of Internal Medicine, Daegu Fatima Hospital, 183 Ayangro, Dong-gu, Daegu 701-600, South Korea
}

incidence of cancer peaks in the seventh decade of human life [6]. Thus, even if resection is possible, surgical treatment cannot be performed in some patients because of their general condition or accompanying comorbidities at the time of diagnosis. In this report, we describe the case of a patient with esophageal cancer who could not undergo surgical treatment because he also had chronic obstructive pulmonary disease (COPD). We successfully treated the patient with endoscopic submucosal dissection (ESD) after concurrent chemoradiotherapy. Our description of the case is accompanied by a discussion of relevant literature.

\section{Case presentation}

An 82-year-old Korean man was admitted to our hospital with a chief complaint of intermittent odynophagia of approximately 3 months' duration. The patient did not have a history of alcohol intake. He was a current 
smoker with a smoking history of 120 pack-years (2 packs daily for 60 years). He had been taking medication and using an inhaler for approximately 20 years to treat his COPD. A pulmonary function test (performed approximately 2 months prior to his presentation) revealed poor respiratory function with a forced expiratory volume in 1 second of approximately $45 \%$.

An endoscopic examination showed a broad, ulcerative lesion at $33 \mathrm{~cm}$ to $38 \mathrm{~cm}$ from the incisor that spanned more than three-fourths of the esophageal lumen circumference (Figures 1A and 1B). The biopsy findings indicated moderately differentiated squamous cell carcinoma. Endoscopic ultrasonography (EUS) revealed a blurring and thickening of the third layer (submucosal layer). However, the fourth layer (proper muscle layer) was spared. In addition, we observed three malignant lymph nodes $>10 \mathrm{~mm}$ that were round in shape, had smooth features and exhibited hypoechogenicity. They were located $27 \mathrm{~cm}, 28 \mathrm{~cm}$ and $42 \mathrm{~cm}$ from the incisor (Figures $2 \mathrm{~A}$, $2 \mathrm{~B}, 2 \mathrm{C}$ and $2 \mathrm{D}$ ). However, we did not find any metastases on a computed tomography (CT) scan or a positron emission tomography (PET)-CT scan.

The patient was clinically diagnosed with T1b N2 M0 G2, stage IIIA cancer (according to the American Joint
Committee on Cancer (AJCC) staging system [7]) and treated with concurrent chemoradiotherapy. His chemotherapy was 5-fluorouracil plus cisplatin, and his radiotherapy of 50 Gy was delivered in 25 fractions. In a follow-up endoscopic examination performed after 5 weeks of chemoradiotherapy, we found that the lesion had decreased in size to approximately one-half the circumference of the esophageal lumen. The ulceration also demonstrated a pattern of improvement upon macroscopic observation (Figures 1C and 1D). In addition, the previously observed malignant lymph nodes were not seen on follow-up EUS, CT and PET-CT scans. After chemoradiotherapy, the patient showed a sufficient response to be clinically down-staged to T1a/T1b N0 M0 G2, stage IA (according to the AJCC staging system [7]). Though additional surgical treatment was considered with the goal of a complete cure, we acquired informed consent for ESD instead after explaining to the patient and his guardian the high post-operative risk posed by his class IV condition (American Society of Anesthesiologists classification), which was due to his poor pulmonary function (Figures $3 \mathrm{~A}, 3 \mathrm{~B}$ and $3 \mathrm{C}$ ). A specimen measuring approximately $5 \times 4.8 \mathrm{~cm}$ in size was acquired through an en bloc resection (Figure 4A). Using the

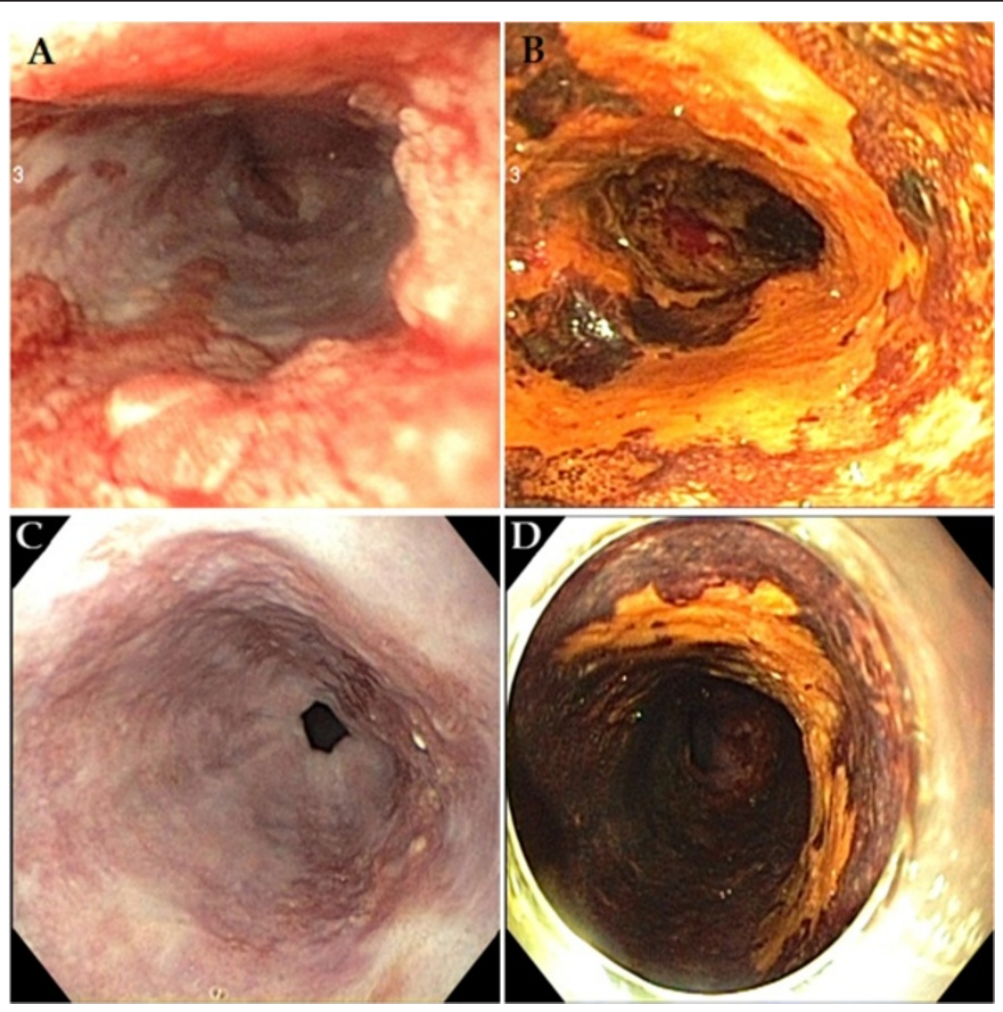

Figure 1 Endoscopic examination. Esophageal cancer was observed at the middle to distal esophagus. It spanned more than three-fourths of the luminal circumference. (A) White light endoscopy. (B) Chemoendoscopy with iodine staining. After chemoradiotherapy, the lesion size decreased to approximately half of the circumference of the esophageal lumen. (C) White light endoscopy. (D) Chemoendoscopy with iodine staining. 

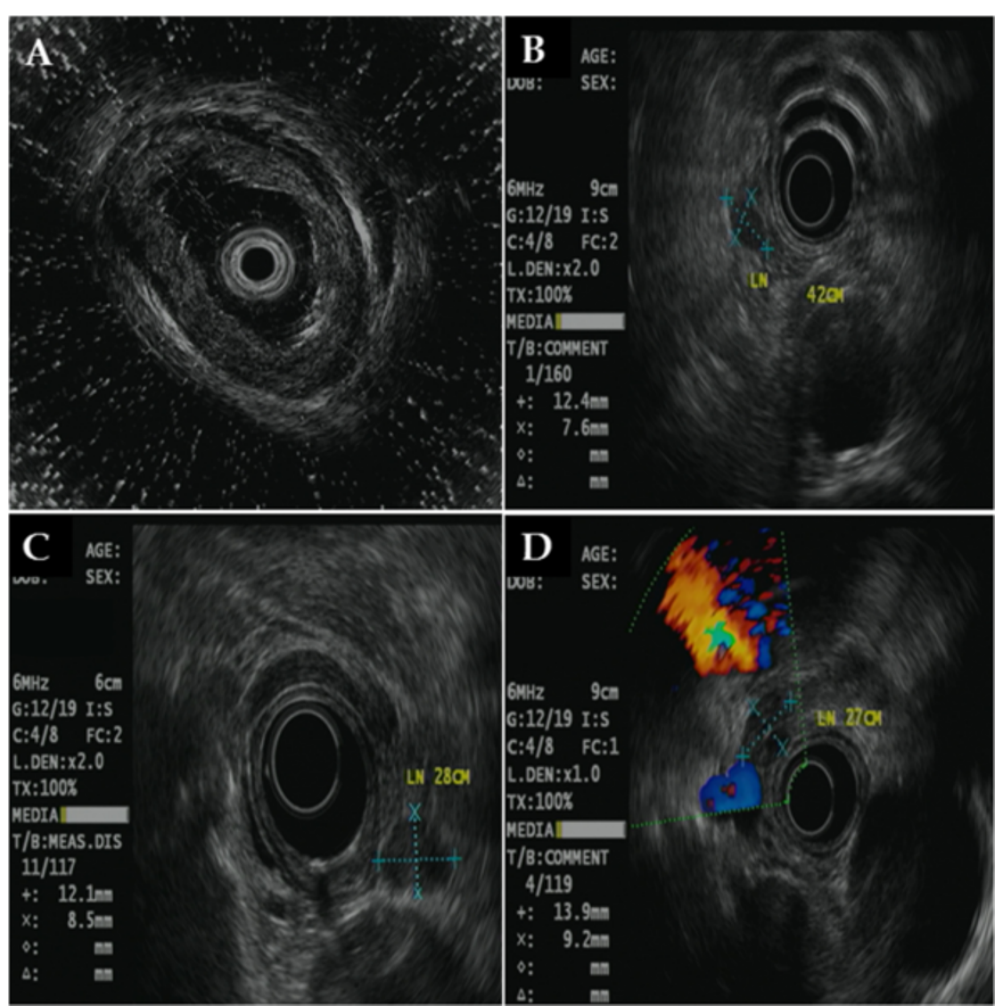

Figure 2 Endoscopic ultrasonography findings. (A) Blurring and thickening of the submucosal layer were observed, but the proper muscle layer was spared. Three enlarged regional lymph nodes $(>10 \mathrm{~mm})$ were observed at $42 \mathrm{~cm}$ (B), $28 \mathrm{~cm}$ (C) and $27 \mathrm{~cm}$ (D) from the incisor.

specimen, we confirmed the diagnosis of moderately differentiated squamous cell carcinoma with focal observation of submucosal invasion (sm1, shallower onethird of the submucosa) (Figure 4B).

After ESD, the patient developed an iatrogenic ulcer covering nearly the total circumference of the esophageal lumen. We therefore predicted a high possibility of secondary luminal stenotic change in the future. Consequently, we administered endoscopic triamcinolone injections (ETIs) on the third, seventh and tenth days after ESD. Follow-up endoscopic examinations were performed at 1 week and at 1, 3 and 6 months after ETI (Figure 5A, 5B, 5C and 5D). They showed neither luminal stenosis nor local recurrence. In addition, esophagography and esophageal manometry tests performed 3 months after ETI did not yield findings of particular interest.

The patient has been followed on an outpatient basis and has not displayed abnormal symptoms such as dysphagia or regurgitation. Currently, neither local nor distant recurrences of esophageal cancer have been observed after 36 months.

The study protocol was approved by Daegu Fatima Hospital Ethics Committee. The patient gave us written informed consent before the procedure.

\section{Discussion}

As is the case with the majority of gastrointestinal tract malignancies, esophageal cancer has a potentially curative therapy consisting of surgical treatment (esophagectomy with therapeutic lymphadenectomy). However, with recent reports of survival benefits [8] and complete resection rate increases in patients receiving neoadjuvant chemoradiotherapy [9], that approach has received attention as a standard treatment for locally advanced esophageal cancer [10]. Our patient had stage IIIA disease at the time of initial diagnosis, but restaging after neoadjuvant chemoradiotherapy showed a significant down-staging effect to stage IA disease.

It is well known that the surgical risk of esophagectomy varies by clinical characteristics such as age and comorbidities. Finlayson et al. reported that the 30-day mortality of esophagectomy was $10.7 \%$ among patients in their 60s, but that it increased to $20 \%$ among patients in their 80s [11]. Moreover, it is well known that the presence and severity of comorbidities affect disease prognosis as well as surgical outcome. Our patient was 82 years old and exhibited poor pulmonary function. Therefore, we could not perform surgical treatment, despite the fact that his esophageal cancer remained in a resectable state, and we performed ESD instead. 


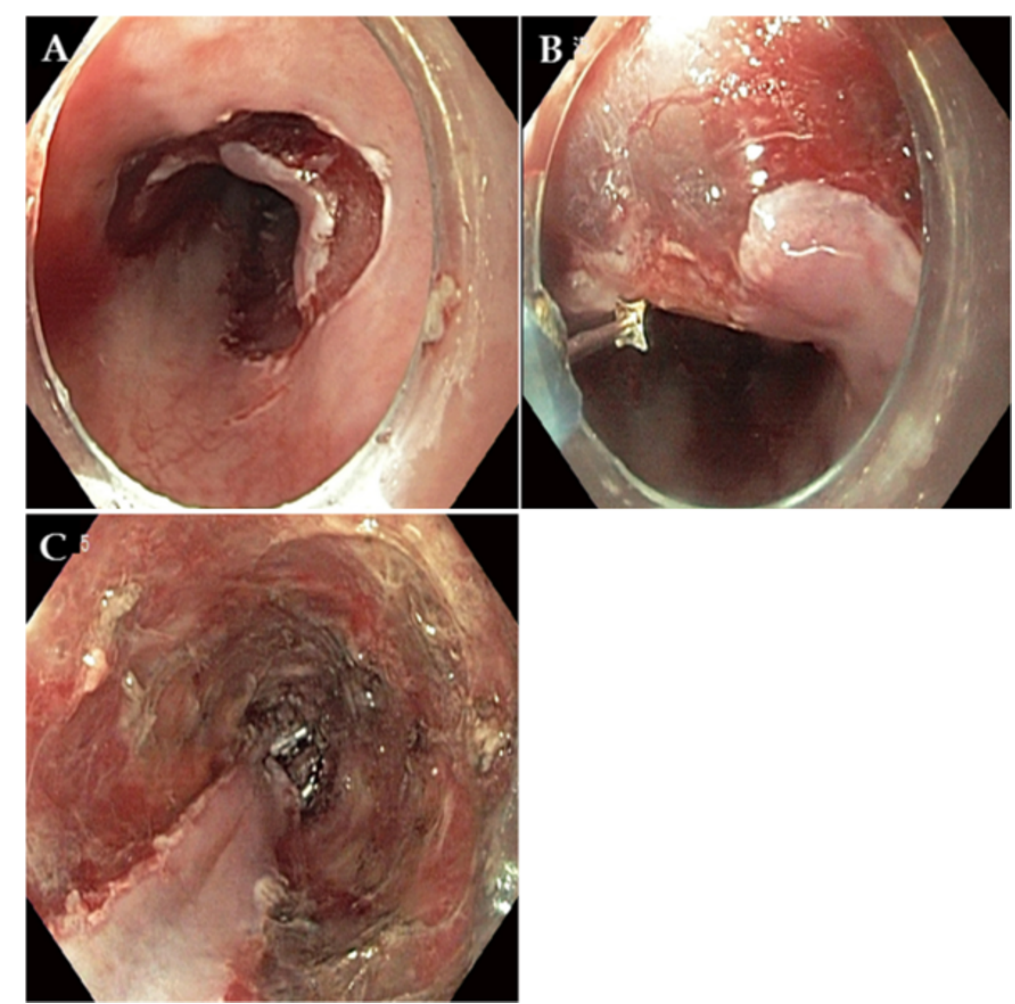

Figure 3 Endoscopic submucosal dissection. (A) Pre-cutting was performed along the outer border of the lesion. (B) Submucosal dissection was performed. (C) A large iatrogenic ulcer was observed after the procedure.

In actuality, endoscopic resection should be avoided in patients with esophageal cancer with submucosal invasion, because there is a substantial risk of regional lymph node metastasis. Ancona et al. reported confirmation of lymph node metastasis in approximately $28 \%$ of esophageal cancers with submucosal layer invasion [12]. In our patient, EUS performed during diagnosis showed invasion of the submucosal layer and three regional metastatic nodes. However, EUS performed after neoadjuvant chemoradiotherapy showed an absence of these regional lymph nodes, and ESD was planned only then. Clearly, ESD would not have been planned as a follow-up treatment if, in the initial diagnosis, there were EUS or CT findings that the lesion had invaded the muscularis propria or adventitia or any adjacent structures. Furthermore, we could not directly confirm $\mathrm{T}$ stage improvement by EUS prior to ESD, because radiation-induced inflammation of the esophageal wall and residual disease cannot be differentiated easily on EUS scans after neoadjuvant chemoradiotherapy [13]. However, at the time of initial

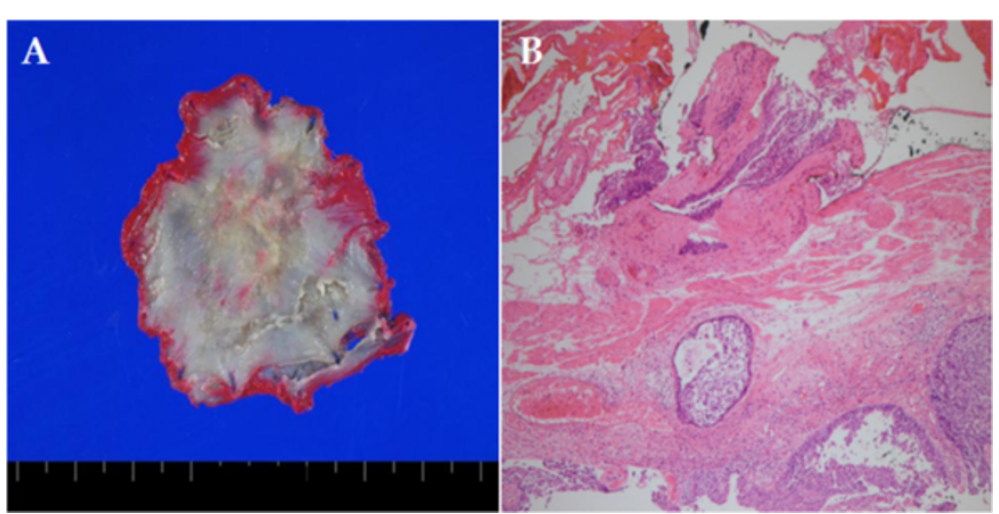

Figure 4 Pathologic findings. (A) Macroscopic finding of a specimen approximately $5 \mathrm{~cm}$ in size after en bloc resection. (B) Squamous cell carcinoma with focal submucosal invasion. 


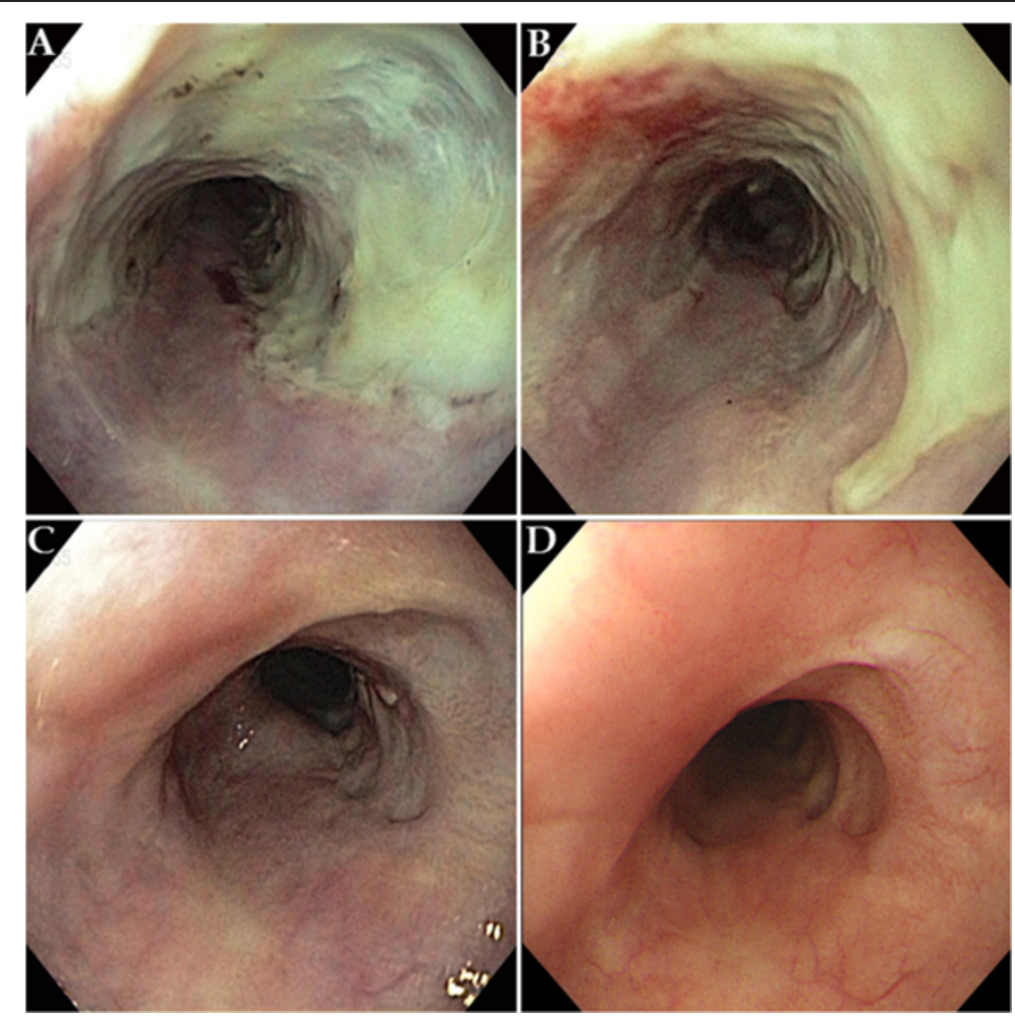

Figure 5 Endoscopic findings after endoscopic triamcinolone injection. (A) 1 week post-injection. (B) 1 month post-injection. (C) 3 months post-injection. (D) 6 months post-injection.

diagnosis, EUS showed only submucosal invasion of the lesion. In addition, as regional lymph nodes showed improvement following neoadjuvant chemoradiotherapy, we indirectly predicted that the $\mathrm{T}$ staging of the lesion would not progress beyond T1b. We therefore thoroughly explained the process to the patient and his guardians and acquired informed consent to proceed with ESD.

After neoadjuvant chemoradiotherapy, the lesion area decreased significantly. However, as we performed ESD with a sufficient lateral safety margin, an iatrogenic ulcer that occupied the majority of the short axis of the esophageal lumen unavoidably developed post-operatively. We therefore performed ETI to prevent a secondary luminal stricture. The ETI was performed following a protocol described by Hashimoto et al. [14]. In accordance with that protocol, triamcinolone acetonide $(10 \mathrm{mg} / \mathrm{ml})$ was placed in a $1-\mathrm{ml}$ syringe. Then, a 25 -gauge, $4 \mathrm{~mm}$ needle (FINEMEDIX, Daegu, South Korea) was used to inject the triamcinolone in increments of $0.2 \mathrm{ml}(2 \mathrm{mg})$ at $1 \mathrm{~cm}$ intervals along the long axis and short axis of the ulcer base [14]. No luminal stricture was observed in a followup endoscopic examination performed after triamcinolone injection.

The patient remained in a disease-free state without local or distant recurrence 36 months after ESD. This is considered to be due to the fact that the cancer differentiation was not "poor" and the regional lymph node metastases resolved as a result of neoadjuvant chemoradiotherapy. The primary cancer site was also successfully treated to R0 resection through ESD and now shows a good prognosis.

\section{Conclusions}

On the basis of our experience in this case, we think that ESD treatment can be considered in patients with resectable esophageal cancer if surgery poses a high risk because of comorbidities or the age of the patient and if there is a good response to neoadjuvant chemoradiotherapy. Furthermore, with respect to the large iatrogenic ulcer that occurred after ESD in our patient, we found that triamcinolone injection via endoscopy was effective in preventing luminal stricture.

\section{Consent}

Written informed consent was obtained from the patient for publication of this case report and any accompanying images. A copy of the written consent is available for review by the Editor-in-Chief of this journal.

\section{Competing interests}

The authors declare that they have no competing interests. 


\section{Authors' contributions}

SAL and GEK were the attending doctors for the patient. JMK was involved in the preparation of the manuscript. JKP helped with the diagnosis and management of the patient. YJC and JKJ administered neoadjuvant chemoradiotherapy. CKP performed endoscopic ultrasonography. HSK designed the report. JHL wrote the manuscript. DWL performed endoscopic submucosal dissection and contributed to revising the manuscript. All authors read and approved the final manuscript.

\section{Acknowledgments}

We thank Man Hoon Han, MD, for his thoughtful insight into the pathology of esophageal cancer.

Received: 10 October 2014 Accepted: 26 October 2014

Published: 18 December 2014

\section{References}

1. Allum WH, Stenning SP, Bancewicz J, Clark PI, Langley RE: Long-term results of a randomized trial of surgery with or without preoperative chemotherapy in esophageal cancer. J Clin Oncol 2009, 27:5062-5067.

2. Ando N, lizuka T, Ide H, Ishida K, Shinoda M, Nishimaki T, Takiyama W, Watanabe $\mathrm{H}$, Isono K, Aoyama N, Makuuchi H, Tanaka O, Yamana H, Ikeuchi S, Kabuto T, Nagai K, Shimada Y, Kinjo Y, Fukuda H, Japan Clinical Oncology Group: Surgery plus chemotherapy compared with surgery alone for localized squamous cell carcinoma of the thoracic esophagus: a Japan Clinical Oncology Group Study-JCOG9204. J Clin Oncol 2003, 21:4592-4596.

3. Zieren HU, Müller JM, Jacobi CA, Pichlmaier H, Müller RP, Staar S: Adjuvant postoperative radiation therapy after curative resection of squamous cell carcinoma of the thoracic esophagus: a prospective randomized study. World J Surg 1995, 19:444-449.

4. Mariette C, Piessen G, Triboulet JP: Therapeutic strategies in oesophageal carcinoma: role of surgery and other modalities. Lancet Oncol 2007, 8:545-553.

5. Sjoquist KM, Burmeister BH, Smithers BM, Zalcberg JR, Simes RJ, Barbour A, Gebski V, Australasian Gastro-Intestinal Trials Group: Survival after neoadjuvant chemotherapy or chemoradiotherapy for resectable oesophageal carcinoma: an updated meta-analysis. Lancet Oncol 2011, 12:681-692.

6. Zhang Y: Epidemiology of esophageal cancer. World J Gastroenterol 2013, 19:5598-5606.

7. Edge S, Byrd DR, Compton CC, Fritz AG, Greene GL, Trotti A: AJCC Cancer Staging Manual. 7th edition. New York: Springer; 2010.

8. Gebski V, Burmeister B, Smithers BM, Foo K, Zalcberg J, Simes J, Australasian Gastro-Intestinal Trials Group: Survival benefits from neoadjuvant chemoradiotherapy or chemotherapy in oesophageal carcinoma: a meta-analysis. Lancet Oncol 2007, 8:226-234.

9. Courrech Staal EFW, Aleman BMP, Boot H, van Velthuysen MLF, van Tinteren $H$, van Sandick JW: Systematic review of the benefits and risks of neoadjuvant chemoradiation for oesophageal cancer. Br J Surg 2010, 97:1482-1496.

10. Stahl M, Stuschke M, Lehmann N, Meyer HJ, Walz MK, Seeber S, Klump B, Budach W, Teichmann R, Schmitt M, Schmitt G, Franke C, Wilke H: Chemoradiation with and without surgery in patients with locally advanced squamous cell carcinoma of the esophagus. J Clin Oncol 2005, 23:2310-2317. A published erratum appears in J Clin Oncol 2006, 24:531.

11. Finlayson EV, Birkmeyer JD: Operative mortality with elective surgery in older adults. Eff Clin Pract 2001, 4:172-177. A published erratum appears in Eff Clin Pract 2001, 4:235.

12. Ancona E, Rampado S, Cassaro M, Battaglia G, Ruol A, Castoro C, Portale G, Cavallin F, Rugge M: Prediction of lymph node status in superficial esophageal carcinoma. Ann Surg Oncol 2008, 15:3278-3288

13. ASGE Standards of Practice Committee, Evans JA, Early DS, Chandraskhara V, Chathadi KV, Fanelli RD, Fisher DA, Foley KQ, Hwang JH, Jue TL, Pasha SF, Sharaf R, Shergill AK, Dominitz JA, Cash BD: The role of endoscopy in the assessment and treatment of esophageal cancer. Gastrointest Endosc 2013, 77:328-334.

14. Hashimoto S, Kobayashi M, Takeuchi M, Sato Y, Narisawa R, Aoyagi Y: The efficacy of endoscopic triamcinolone injection for the prevention of esophageal stricture after endoscopic submucosal dissection. Gastrointest Endosc 2011, 74:1389-1393.

doi:10.1186/1752-1947-8-439

Cite this article as: Lim et al.: Esophageal cancer treated by endoscopic submucosal dissection after neoadjuvant chemoradiotherapy: a case report. Journal of Medical Case Reports 2014 8:439.

\section{Submit your next manuscript to BioMed Central and take full advantage of:}

- Convenient online submission

- Thorough peer review

- No space constraints or color figure charges

- Immediate publication on acceptance

- Inclusion in PubMed, CAS, Scopus and Google Scholar

- Research which is freely available for redistribution

Submit your manuscript at www.biomedcentral.com/submit
C Biomed Central 